\title{
Enhancing interaction in maritime spatial planning workshops through collaborative GIS
}

\author{
Mikko Rönneberg $^{\mathrm{a}, *}$, Christian Koski ${ }^{\text {a }}$, Pyry Kettunen ${ }^{\text {a }}$, Juha Oksanen ${ }^{\text {a }}$ \\ ${ }^{a}$ Finnish Geospatial Research Institute FGI, Mikko Rönneberg-mikko.rönneberg@nls.fi, Christian Koski-christian.koski@nls.fi, \\ Pyry Kettunen - pyry.kettunen@nls.fi, Juha Oksanen-juha.oksanen@nls.fi
}

* Corresponding author

Keywords: Collaborative GIS, Maritime Spatial Planning, Baltic Explorer

\begin{abstract}
:
We have created a cross-platform web-map CGIS for collaborative Maritime Spatial Planning (MSP) workshops, called Baltic Explorer [1]. MSP requires simple and easy to use tools for facilitating discussion and managing spatial data in collaborative workshops (Pınarbaşı et al. 2017). Yet, according to reports from real-world MSP workshops, digital spatial tools are used scarcely, with planners preferring paper maps and pens to handle the geospatial interaction. Collaborative GIS (CGIS) offers tools for structuring participation in group spatial decision processes (Balram \& Dragicevic 2006). Therefore, CGIS can have potential to improve many aspects of MSP workshops.

Baltic Explorer offers multi-user map workspaces for collaborative geospatial data exploration, spatial analysis and collaborative map drawing, Figure 1. In MSP workshops the workflow with Baltic Explorer starts with organisers creating workspaces for the participants. During the workshop participants can explore the large amount of MSP related geospatial data overlays provided by multiple SDIs. This offers overall and detailed insight for the workshop task. Participants can contribute by sharing features they have drawn on the workspace map. These features can be viewed and edited by other participants to allow for a more interactive way of collaborating.
\end{abstract}

Baltic Explorer was demonstrated and evaluated in two real-word MSP workshops as well as in a gamified user test. The first workshop was a cross-border meeting where various stakeholders discussed planning objectives and available data. The evaluation focused on exploration of geospatial data and collaborative map drawing tools. The second workshop was a cross-sector event where participants were tasked to identify a new suitable wind park site using the on-the-fly spatial analysis tool which was also the focus of the evaluation. In the gamified user test participants were tasked to play a game that simulated common MSP workshop tasks using Baltic Explorer with personal and shared device setups. The evaluation focused on the device setup to be used with CGIS tools in MSP. In all three demonstrations evaluation was carried out via naturalistic observation and questionnaires.

The new way of working with spatial data in MSP workshops was well received by participants. According to the questionnaire and observations in the first workshop, participants found the geospatial data exploration and collaborative map drawing tools helpful for various tasks. The same applied in the second evaluation while also the analysis tool was considered useful. The gamified user test revealed that multiple device types, such as laptops and large screens, are recommended for MSP workshops. Access to geospatial data was considered important in all the workshops. The observation, feedback, results from questionnaires and requirements from literature were used to refine the concept of Baltic Explorer, as well as to design, enhance and add utility to the system (e.g. spatial analysis). The conducted research adds to the knowledge about interaction with spatial data in collaborative workshops, which can enhance future CGIS. 


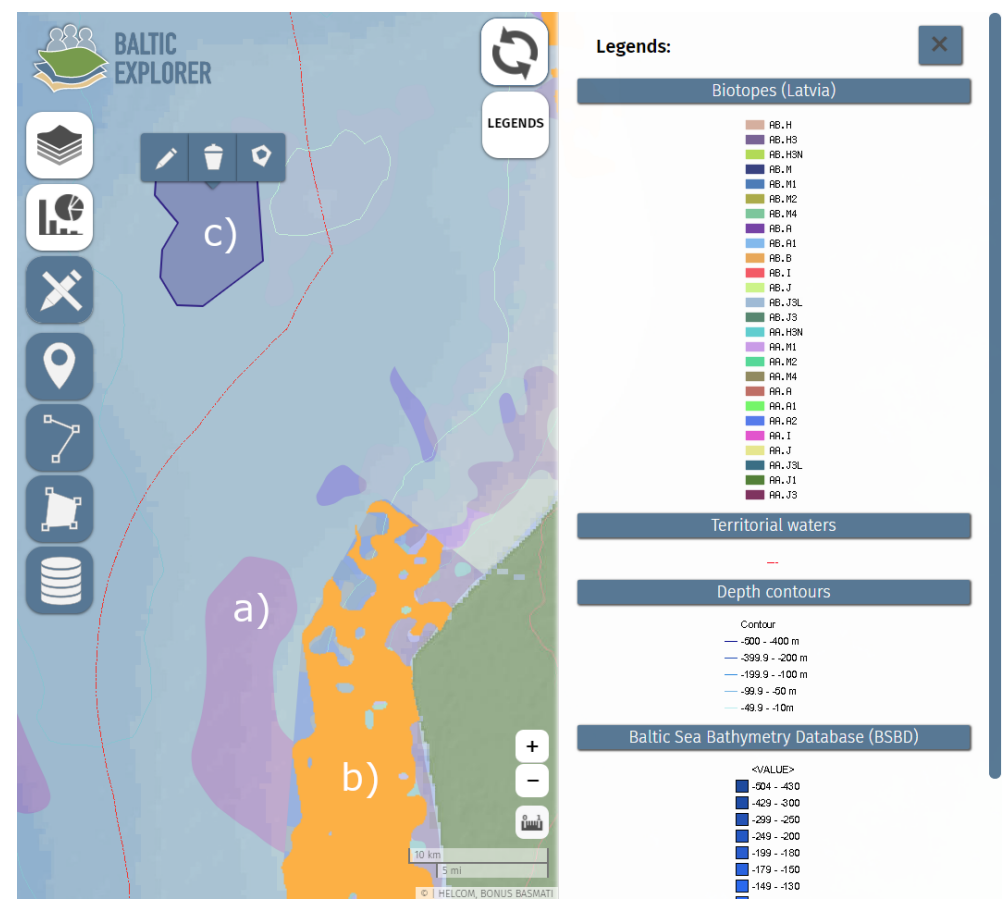

Figure 1. Exploration of geospatial data (a), spatial analysis (b) and collaborative map drawing (c) were considered useful functionalities of Baltic Explorer in a maritime spatial planning workshop for finding a suitable area for a new wind power park (c).

\section{Acknowledgements}

We want to thank all the organisers and participants of the MSP workshops and the user test for their contribution. The BONUS BASMATI project has received funding from BONUS (Art 185), funded jointly by the EU and Innovation Fund Denmark, Swedish Research Council Formas, Academy of Finland, Latvian Ministry of Education and Science, and Forschungszentrum Jülich GmbH (Germany). We made use of geospatial computing resources provided by CSC and the Open Geospatial Information Infrastructure for Research (oGIIR, urn:nbn:fi:research-infras-2016072513) funded by the Academy of Finland.

\section{References}

Balram, S., \& Dragicevic, S. (2006). Collaborative geographic information systems: origins, boundaries, and structures. In Collaborative geographic information systems (pp. 1-23). IGI Global.

Pınarbaşı, K., Galparsoro, I., Borja, Á., Stelzenmüller, V., Ehler, C. N., \& Gimpel, A. (2017). Decision support tools in marine spatial planning: present applications, gaps and future perspectives. Marine Policy, 83, 83-91.

\section{Web Links}

[1] http://balticexplorer.eu 